\title{
Quantum transport using the Ford-Kac-Mazur formalism
}

\author{
Abhishek Dhar ${ }^{1, *}$ and B. Sriram Shastry ${ }^{2, \dagger}$ \\ ${ }^{1}$ Physics Department, University of California, Santa Cruz, California 95064 \\ ${ }^{2}$ Indian Institute of Science, Bangalore 560012, India
}

\begin{abstract}
The Ford-Kac-Mazur formalism is used to study quantum transport in (1) electronic and (2) harmonic oscillator systems connected to general reservoirs. It is shown that for noninteracting systems the method is easy to implement and is used to obtain many exact results on electrical and thermal transport in onedimensional disordered wires. Some of these have earlier been obtained using nonequilibrium Green function methods. We examine the role that reservoirs and contacts can have on determining the transport properties of a wire and find several interesting effects.
\end{abstract}

\section{INTRODUCTION}

There is considerable current interest in the problem of transport through various nanoscale devices both from the fundamental and from applied points of view. In this connection, Kubo's transport formulas have to a large extent been superseded by different formalisms in the spirit of Bardeen's tunneling model. ${ }^{1}$ The Landauer formula ${ }^{2}$ (LF) and the Keldysh technique, ${ }^{3}$ quantum Langevin equations, ${ }^{4} C^{*}$ algebraic formulas, ${ }^{5}$ and generalized scattering theory ideas ${ }^{6}$ have been developed, allowing one to study systems in steady state arbitrarily far from the linear region where $\mathrm{Ku}$ bo's formula is applicable. There is also considerable experimental activity involving resistive elements, such as quantum dots, scanning tunneling microscopy (STM) tips, singlewalled nanotubes, and insulating nanowires, often coming up with unexpected physics. ${ }^{7-9}$

The most popular alternative to Kubo's formulas is the LF, proposed in $1957 .{ }^{2}$ Since then, several derivations of the LF have been given ${ }^{10}$ and this has led to a good understanding of the formula. A large number of experiments are interpreted successfully on the basis of the LF. The quantum of conductance $e^{2} / h$ has been understood as a contact resistance which arises due to the squeezing of the reservoir degrees of freedom into a single channel. ${ }^{11,12}$ While a physically careful statement of the conditions for validity of the LF can be found in Ref. 13, we believe that a detailed mathematical theory of the role of reservoirs and the nature of the coupling between the wires and reservoirs does not exist. The role of the idealized reservoirs has been to serve as perfect sources and sinks of thermal electrons. This clearly will not be satisfied in all experimental conditions, and it is necessary to have a better microscopic understanding of reservoirs and contacts. There has been some work w $^{3,5,6,12,14}$ in this direction but, to our knowledge, a detailed understanding of the role of reservoirs is still lacking.

In this paper we adapt a formalism that was developed by Ford, Kac, and Mazur ${ }^{15}$ (FKM) and model reservoirs as infinite noninteracting systems. This method was originally devised to study Brownian motion in coupled oscillators ${ }^{15}$ and was later extended to a general study of the problem of a quantum particle coupled to a quantum mechanical heat bath. ${ }^{16}$ In this approach reservoirs are modeled by a collec- tion of oscillators which are initially in equilibrium. The reservoir degrees of freedom are then eliminated, leading to quantum Langevin equations for the remaining degrees of freedom (the system). Thus the reservoirs can be viewed as providing sources of noise and dissipation into the system. The FKM formalism is thus very direct to interpret and, as we shall demonstrate, is more straightforward to apply than other methods of treating open quantum systems such as the Caldeira-Leggett, ${ }^{17}$ Keldysh, ${ }^{18}$ and scattering theories. ${ }^{6}$ Quantum Langevin equations have earlier been used in the context of transport in mesoscopic systems and have helped in the understanding of some experimental data. ${ }^{4,19}$ The FKM approach was also used earlier by O'Connor and Lebowitz $^{20}$ in studying classical heat transport in disordered harmonic chains and our analysis here closely follows theirs. Here we use the FKM approach to make a detailed study of quantum transport in disordered electronic and phononic systems. For very general reservoirs we obtain exact formal expressions for currents and local densities in the nonequilibrium steady state. We find that for a special type of reservoir, the ideal Landauer result (where the conductance is expressed in terms of the transmission coefficient of onedimensional plane waves) follows exactly, while for general reservoirs they need to be modified. We examine in some detail the effect on transport properties that the choice of reservoirs can have and find a number of interesting effects. For example in the electron case we find that imperfect contacts can lead to an enhancement of conductivity. In the phonon case we find the surprising result, earlier noted for classical systems, that the heat current $J$ in a long disordered wire decays with system size $N$ as $J \sim 1 / N^{\alpha}$ where $\alpha$ depends on the low-frequency spectral properties of the reservoirs.

The paper is organized as follows: In Sec. I we present the formalism and results for transport in the one-dimensional (1D) Anderson model. In Sec. II we present the formalism and results for transport in disordered harmonic chains. We end with a discussion in Sec. III.

\section{TRANSPORT IN THE ONE-DIMENSIONAL ANDERSON MODEL}

\section{A. Formalism and main results}

The setup: we wish to study conduction in a disordered fermionic system connected to heat and particle reservoirs 


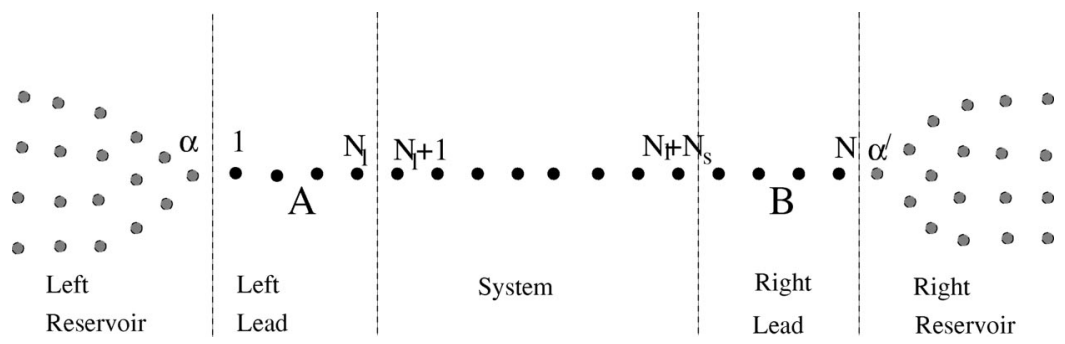

FIG. 1. A disordered system connected through 1D leads to reservoirs at different potentials and temperatures.

through ideal 1D leads (see Fig. 1). We consider a tightbinding model, and for simplicity we take the system and leads to be $1 \mathrm{D}$ while the reservoirs are quite general. We use the following notation: the indices $l, m$ denote points on the system or leads, greek indices $\lambda, \nu$ or $\lambda^{\prime}, \mu^{\prime}$ denote points on the left or right reservoirs, respectively, and finally $p, q$ denote points anywhere. Thus $c_{l}(l=1,2, \ldots, N)$ denotes lattice fermionic operators on the (system + lead), and $c_{\lambda}, c_{\lambda^{\prime}}\left(\lambda, \lambda^{\prime}=1,2, \ldots, M\right)$ denotes operators on the left and right reservoirs. The $c_{p} \mathrm{~s}^{\prime}$ satisfy the usual anticommutation relations $\left\{c_{p}, c_{q}\right\}=0,\left\{c_{p}^{\dagger}, c_{q}^{\dagger}\right\}=0$, and $\left\{c_{p}^{\dagger}, c_{q}\right\}=\delta_{p q}$. Out of the $N=N_{s}+2 N_{l}$ sites, the first and last $N_{l}$ sites refer to the leads while the middle $N_{s}$ sites refer to the system. The Hamiltonian for the entire system is given by $H=H^{0}+V$ $+V_{\text {int }}$, where

$$
\begin{aligned}
H^{0}= & -\sum_{l=1}^{N-1}\left(c_{l}^{\dagger} c_{l+1}+c_{l+1}^{\dagger} c_{l}\right)+\sum_{l=1}^{N} v_{l} c_{l}^{\dagger} c_{l} \\
& +\sum_{\lambda \nu} \hat{T}_{\lambda \nu} c_{\lambda}^{\dagger} c_{\nu}+\sum_{\lambda^{\prime} \nu^{\prime}} \hat{T}_{\lambda^{\prime} \nu^{\prime}}^{\prime} c_{\lambda^{\prime}}^{\dagger} c_{\nu^{\prime}} ; \\
V=- & \gamma\left(c_{1}^{\dagger} c_{\alpha}+c_{\alpha}^{\dagger} c_{1}\right)-\gamma^{\prime}\left(c_{N^{\prime}}^{\dagger} c_{\alpha^{\prime}}+c_{\alpha^{\prime}}^{\dagger} c_{N}\right) .
\end{aligned}
$$

The first part of $H^{0}$ refers to the system and leads, while $\hat{T}$ and $\hat{T}^{\prime}$ describe the two reservoirs. The contact between the reservoirs and leads is given by the interconnection part $V$. The interaction part $V_{\text {int }}$ can be added perturbatively, and we return to its inclusion later in the paper. We will consider a system with on-site disorder and so choose the on-site energies $v_{l}, l=N_{l}+1, \ldots, N_{l}+N_{s}$, from some random distribution. At sites belonging to the leads $\left[l=1,2, \ldots, N_{l}, N_{l}\right.$ $\left.+N_{s}+1, \ldots, N\right]$, assumed to be perfect conductors, we set $v_{l}=0$. At some time $t<\tau$ in the remote past, the two reservoirs are isolated and in equilibrium at chemical potentials $\mu$ and $\mu^{\prime}$ and inverse temperatures $\beta$ and $\beta^{\prime}$, respectively. At $t=\tau$, we connect the reservoirs to the two leads and evolve the system with the Hamiltonian $H$. We study the properties of the nonequilibrium steady state, reached after a long time.

The Heisenberg equations of motion for the operators of the system and leads are given by (for $t>\tau$ )

$$
\begin{gathered}
\dot{c}_{1}=i c_{2}-i v_{1} c_{1}+i \gamma c_{\alpha}, \\
\dot{c}_{l}=i\left(c_{l-1}+c_{l+1}\right)-i v_{l} c_{l}(2 \leqslant l \leqslant N-1), \\
\dot{c}_{N}=i c_{N-1}-i v_{N} c_{N}+i \gamma^{\prime} c_{\alpha^{\prime}} .
\end{gathered}
$$

The equations at the boundary sites involve reservoir operators $c_{\alpha}$ and $c_{\alpha^{\prime}}$. Using the equations of motion of the reservoir variables we can replace these reservoir operators by Langevin-type terms. The equations for the left reservoir are given by (for $t>\tau$ )

$$
\begin{gathered}
\dot{c}_{\lambda}=-i T_{\lambda \nu} c_{\nu}(\lambda \neq \alpha), \\
\dot{c}_{\alpha}=-i T_{\alpha \nu} c_{\nu}+i \gamma c_{1} .
\end{gathered}
$$

This is a linear set of equations with an inhomogeneous part given by the term $i \gamma c_{1}$ and has the general solution

$$
c_{\lambda}(t)=i \sum_{\nu} g_{\lambda \nu}^{+}(t-\tau) c_{\nu}(\tau)-\int_{\tau}^{\infty} d t^{\prime} g_{\lambda \alpha}^{+}\left(t-t^{\prime}\right)\left[\gamma c_{1}\left(t^{\prime}\right)\right]
$$

where

$$
g_{\lambda \nu}^{+}(t)=-i \theta(t) \sum_{n} \psi_{n}(\lambda) \psi_{n}^{*}(\nu) e^{-i \epsilon_{n} t}
$$

Here $\psi_{n}(\lambda)$ is the single-particle eigenstate of the left reservoir, with energy $\epsilon_{n}$, and $n$ runs over all states. We need $c_{\alpha}(t)$ which we note has two parts. The first, $h(t)$ $=i \Sigma_{\nu} g_{\alpha \nu}^{+}(t-\tau) c_{\nu}(\tau)$, is like a noise term whose statistics is determined by the initial conditions of the reservoir. Initially the reservoirs are in thermal equilibrium and the normal modes $\quad c_{n}=\Sigma_{\lambda} c_{\lambda} \psi_{n}(\lambda) \quad$ satisfy $\left\langle c_{n}^{\dagger}(\tau) c_{n^{\prime}}(\tau)\right\rangle$ $=\delta_{n n^{\prime}} f\left(\epsilon_{n}, \mu, \beta\right)$, where $f$ is the Fermi distribution, $f$ $=1 /\left[e^{\beta\left(\epsilon_{n}-\mu\right)}+1\right]$, and $\langle\hat{O}\rangle=\operatorname{Tr}[\hat{O} \hat{\rho}]$, where $\hat{\rho}$ is the reservoir density matrix at time $\tau$ and "Tr" denotes a trace over reservoir variables. The second part of $c_{\alpha}(t)$, $c_{\alpha}(t), \quad-\gamma \int_{\tau}^{\infty} d t^{\prime} g_{\alpha \alpha}^{+}\left(t-t^{\prime}\right) c_{1}\left(t^{\prime}\right)$, is dissipative in nature. Defining the Fourier transforms $c_{p}(\omega)$ $=(1 / 2 \pi) \int_{-\infty}^{\infty} d t c_{p}(t) e^{i \omega t}, \quad g_{\alpha \alpha}^{+}(\omega)=\int_{-\infty}^{\infty} d t g_{\alpha \alpha}^{+}(t) e^{i \omega t}$, and $h(\omega)=(1 / 2 \pi) \int_{-\infty}^{\infty} d t h(t) e^{i \omega t}$, and taking the limits $M \rightarrow \infty$ and $\tau \rightarrow-\infty$, we get

$$
\begin{gathered}
c_{\alpha}(\omega)=h(\omega)-\gamma g_{\alpha \alpha}^{+}(\omega) c_{1}(\omega), \\
\left\langle h^{\dagger}(\omega) h\left(\omega^{\prime}\right)\right\rangle=I(\omega) \delta\left(\omega-\omega^{\prime}\right), \\
I(\omega)=\rho_{\alpha}(\omega) f(\omega), \\
g_{\alpha \alpha}^{+}(\omega)=\sum_{n} \frac{\left|\psi_{n}(\alpha)\right|^{2}}{\omega-\epsilon_{n}}-i \pi \rho_{\alpha},
\end{gathered}
$$

where $\rho_{\alpha}=\Sigma_{n}\left|\psi_{n}(\alpha)\right|^{2} \delta\left(\omega-\epsilon_{n}\right)$ is the density of states at site $\alpha$. The third equation above is a statement of the fluctuation-dissipation theorem. Similarly for the right reser- 
voir we get $c_{\alpha^{\prime}}(\omega)=h^{\prime}(\omega)-\gamma^{\prime} g_{\alpha^{\prime} \alpha^{\prime}}^{+}(\omega) c_{N}(\omega)$, with the noise statistics of $h^{\prime}(\omega)$ determined by $\mu^{\prime}$ and $\beta^{\prime}$. Also $h$ and $h^{\prime}$ are independent so that $\left\langle h^{\dagger}(\omega) h^{\prime}\left(\omega^{\prime}\right)\right\rangle=0$. We now Fourier transform the system equations and plug in the forms of $c_{\alpha}(\omega)$ and $c_{\alpha^{\prime}}(\omega)$ to get the following particular solution:

$$
\begin{gathered}
c_{l}(t)=\int_{-\infty}^{\infty} d \omega \hat{Z}_{l m}^{-1}(\omega) h_{m}(\omega) e^{-i \omega t}, \\
\hat{Z}_{l m}=\hat{\Phi}_{l m}+\hat{A}_{l m}, \\
\hat{\Phi}_{l m}=-\delta_{l, m+1}-\delta_{l, m-1}+\left(v_{l}-\omega\right) \delta_{l, m}, \\
\hat{A}_{l m}=\delta_{l, m}\left[\gamma^{2} g_{\alpha \alpha}^{+}(\omega) \delta_{l, 1}+\gamma^{\prime 2} g_{\alpha^{\prime} \alpha^{\prime}}^{+}(\omega) \delta_{l, N}\right], \\
h_{l}=\gamma h(\omega) \delta_{l, 1}+\gamma^{\prime} h^{\prime}(\omega) \delta_{l, N} .
\end{gathered}
$$

With this formal solution and the known properties of the spectral functions $h(\omega), h^{\prime}(\omega), g_{\alpha \alpha}^{+}(\omega)$, and $g_{\alpha^{\prime} \alpha^{\prime}}^{+}(\omega)$, we can now compute various physical quantities of interest. Specifically we shall be interested in the electrical and thermal currents and the local particle and energy densities. The operators corresponding to particle and energy densities are given by

$$
\begin{gathered}
\hat{n}_{l}=c_{l}^{\dagger} c_{l}, \\
\hat{u}_{l}=-\left(c_{l}^{\dagger} c_{l+1}+c_{l+1}^{\dagger} c_{l}\right) \\
+\frac{1}{2}\left(v_{l} c_{l}^{\dagger} c_{l}+v_{l+1} c_{l+1}^{\dagger} c_{l+1}\right),
\end{gathered}
$$

while the corresponding current operators $\hat{j}^{n}$ and $\hat{j}^{u}$ are defined through the conservation equations $\partial \hat{n} / \partial t+\partial \hat{j}^{n} / \partial x=0$ and $\partial \hat{u} / \partial t+\partial \hat{j}^{u} / \partial x=0$. We get

$$
\begin{gathered}
\hat{j}_{l}^{n}=i\left(c_{l+1}^{\dagger} c_{l}-c_{l}^{\dagger} c_{l+1}\right), \\
\hat{j}_{l}^{u}=-i\left(c_{l+2}^{\dagger} c_{l}-c_{l}^{\dagger} c_{l+2}\right)+\frac{v_{l+1}}{2}\left(\hat{j}_{l+1}^{n}+\hat{j}_{l}^{n}\right) .
\end{gathered}
$$

We now calculate the steady-state averages of these four quantities. We introduce some notation and state a few mathematical identities. We denote by $Y_{l, m}$ the determinant of the submatrix of $\hat{Z}$ beginning with the $l$ th row and column and ending with the $m$ th row and column. Similarly $D_{l, m}$ denotes determinant of the submatrix formed from $\hat{\Phi}$. The following results can be proved: (i) $Y_{1, N}=D_{1, N}+\gamma^{2} g_{\alpha \alpha}^{+} D_{2, N}$ $+\gamma^{\prime 2} g_{\alpha^{\prime} \alpha^{\prime}}^{+} D_{1, N-1}+\gamma^{2} \gamma^{\prime 2} g_{\alpha \alpha}^{+} g_{\alpha^{\prime} \alpha^{\prime}}^{+} D_{2, N-1}$, (ii) $\hat{Z}_{l N}^{-1}$ $=Y_{1, l-1} / Y_{1, N}, \quad \hat{Z}_{l 1}^{-1}=Y_{l+1, N} / Y_{1, N}$, and (iii) $D_{1, n-1} D_{2, n}$ $-D_{1, n} D_{2, n-1}=1$.

\section{Particle and heat currents}

The expectation value of the current operators, using Eqs. (3) and (21), gives

$$
\begin{gathered}
\left\langle j_{l}^{n}\right\rangle=-2 \int_{-\infty}^{\infty} d \omega \operatorname{Im}\left[\sum_{r=1, N} \hat{Z}_{l+1, r}^{-1 *}(\omega) \hat{Z}_{l, r}^{-1}(\omega) I_{r}(\omega)\right], \\
\left\langle\hat{j}_{l}^{u}\right\rangle=2 \int_{-\infty}^{\infty} d \omega \operatorname{Im}\left[\sum_{r=1, N} \hat{Z}_{l+2, r}^{-1 *}(\omega) \hat{Z}_{l, r}^{-1}(\omega) I_{r}(\omega)\right],
\end{gathered}
$$

where $I_{1}=\gamma^{2} I$ and $I_{N}=\gamma^{\prime 2} I^{\prime}$. In the case of the heat current we take $l$ to be on the leads so that $v_{l}=0$. Using the various identities stated earlier we can show, as expected, that these are independent of $l$ and reduce to the simpler expressions

$$
\begin{gathered}
\left\langle\hat{j}_{l}^{n}\right\rangle=\int_{-\infty}^{\infty} d \omega J(\omega)\left[f(\omega)-f^{\prime}(\omega)\right], \\
\left\langle\hat{j}_{l}^{u}\right\rangle=\int_{-\infty}^{\infty} d \omega \omega J(\omega)\left[f(\omega)-f^{\prime}(\omega)\right],
\end{gathered}
$$

where

$$
J(\omega)=2 \pi \gamma^{2} \gamma^{\prime 2} \rho_{\alpha}(\omega) \rho_{\alpha^{\prime}}(\omega) /\left|Y_{1, N}\right|^{2} .
$$

These can be expressed in terms of the retarded Green's function $G^{+}(\omega)=(\omega+i \epsilon-H)^{-1}$. This satisfies $G^{+}=g^{+}$ $+g^{+} V G^{+}$where $g^{+}=\left(\omega+i \epsilon-H^{0}\right)^{-1}$. These can be solved to give

$$
\begin{gathered}
G_{1 m}^{+}=\left[g_{1 m}^{+}-\gamma^{\prime 2} g_{\alpha^{\prime} \alpha^{\prime}}^{+}\left(g_{N N}^{+} g_{1 m}^{+}-g_{1 N}^{+} g_{N m}^{+}\right)\right] / Z, \\
G_{N m}^{+}=\left[g_{N m}^{+}-\gamma^{2} g_{\alpha \alpha}^{+}\left(g_{11}^{+} g_{N m}^{+}-g_{N 1}^{+} g_{1 m}^{+}\right)\right] / Z,
\end{gathered}
$$

where

$$
\begin{aligned}
Z= & 1-\gamma^{2} g_{11}^{+} g_{\alpha \alpha}^{+}-\gamma^{\prime 2} g_{N N}^{+} g_{\alpha^{\prime} \alpha^{\prime}}^{+} \\
& +\gamma^{2} \gamma^{\prime 2} g_{\alpha \alpha}^{+} g_{\alpha^{\prime} \alpha^{\prime}}^{+}\left(g_{11}^{+} g_{N N}^{+}-g_{1 N}^{+} g_{N 1}^{+}\right) .
\end{aligned}
$$

Let $g_{l m}=\operatorname{Re}\left[g_{l m}^{+}\right]$denote the real part of the system's Green function. It is easy to see that $g_{1 N}=g_{N 1}=-1 / D_{1, N}$, $g_{11}=-D_{2, N} / D_{1, N}$, and $g_{N N}=-D_{1, N-1} / D_{1, N}$. Using these and the Jacobi identity $g_{11} g_{N N}-g_{1 N} g_{N 1}=D_{2, N-1} / D_{1, N}$ we get $1 /\left|Y_{1, N}\right|^{2}=G n_{1 N}^{+} G n_{N 1}^{-}$where $G n^{+}$is a modified Green function obtained from $G^{+}$by replacing all system Green functions by their real part. We then get the particle current in a form similar to those obtained by Meir and Wingreen ${ }^{14}$ using the Keldysh formalism and by Todorov et al. ${ }^{6}$ using time-independent scattering theory. Their results differ from ours in that they are expressed in terms of $G^{+}$instead of $\mathrm{Gn}^{+}$. The case of insulating wires treated by Caroli et $a .^{3}$ also follows from our results.

\section{Scattering states}

It is instructive to write the currents and densities in terms of properties of the single-particle scattering states of the full Hamiltonian $H$ (possible when interactions are absent). Let $\psi^{j L}(\omega)$ and $\psi^{j R}(\omega)$ denote the $j$ th unperturbed wave functions with energy $\omega$ of the left and right reservoirs, respectively. Let $a_{p}^{j L}$ and $a_{p}^{j R}$ denote the amplitude at site $p$ of the $j$ th right- and left-moving states obtained by evolving the unperturbed levels with the full Hamiltonian. We then get 
$a_{l}^{j L}=K_{l 1}^{-1} \gamma \psi_{\alpha}^{j L}(\omega)$ and $a_{l}^{j R}=K_{l N}^{-1} \gamma^{\prime} \psi_{\alpha^{\prime}}^{j R}(\omega)$. The currents and densities are given by $j_{l}^{n}=i\left(a_{l+1}^{*} a_{l}-a_{l}^{*} a_{l+1}\right), n_{l}$ $=a_{l}^{*} a_{l}$, etc. Using these we find that $J(\omega)$ is simply the total transmitted current for all waves with energy $\omega$. Also the particle density is given by

$$
\left\langle\hat{n}_{l}\right\rangle=\int_{-\infty}^{\infty} d \omega\left[\rho_{l}^{L}(\omega) f(\omega)+\rho_{l}^{R}(\omega) f^{\prime}(\omega)\right],
$$

where $\rho_{l}^{L}=\Sigma_{j}\left|a_{l}^{j L}\right|^{2}$ is the total particle density at a point $l$ due to all right-moving waves with energy $\omega$ and $\rho_{l}^{R}$ is due to left movers. Note that the currents and densities do not have the simple Landauer form since $J(\omega)$ depends not only on the system but also on bath and contact properties. The spectral properties of the baths enter into the expressions in a nontrivial way and one cannot separate the contributions of the system and the baths.

\section{B. Ideal reservoirs and contacts: The Landauer case}

This corresponds to the case where $\gamma=\gamma^{\prime}=1$ and the reservoirs themselves are semi-infinite extensions of the onedimensional leads. This results in reflectionless contacts between the reservoirs and leads. The reservoir wave functions and energy eigenvalues are $\psi_{n}(\lambda)=[2 /(M+1)]^{1 / 2} \sin (k \lambda)$ and $\epsilon_{n}=-2 \cos (k)$ where $k=n \pi /(M+1)$ with $n$ $=1,2, \ldots, M$. The leads are connected at the end of the reservoir chains so that $\alpha=\alpha^{\prime}=1$. We then get the following reservoir spectral functions:

$$
\begin{gathered}
I(\omega)=\frac{1}{\pi}\left[1-w^{2} / 4\right]^{1 / 2} f(\omega, \mu, \beta), \quad|\omega|<2, \\
I(\omega)=0, \quad|\omega|>2, \\
g_{\alpha \alpha}^{+}(\omega)=-e^{i k}, \quad-2 \leqslant \omega=-2 \cos (k) \leqslant 2, \\
=\omega / 2-\operatorname{sgn}(\omega)\left(\omega^{2} / 4-1\right)^{1 / 2}, \quad|\omega|>2 .
\end{gathered}
$$

We have similar expressions for the right reservoir. Let us use the notation that if sites $N_{l}+l$ and $N_{l}+m$ belong to the system, then we write $Y_{N_{l}+l, N_{l}+m}=y_{l, m}$ and $D_{N_{l}+l, N_{l}+m}$ $=d_{l, m}$. It can be shown that the transmission probability of a wave with momentum $k$ across the system is given by

$$
T=\frac{4 \sin ^{2}(k)}{\left|y_{1, N_{s}}\right|^{2}}
$$

where

$$
\left|y_{1, N_{s}}\right|=\left|d_{1, N_{s}}-e^{i k}\left(d_{2, N_{s}}+d_{1, N_{s}-1}\right)+e^{i 2 k} d_{2, N_{s}-1}\right| .
$$

Note that in this case the transmission factor does not involve properties of the reservoirs and contacts. Also transmission is only by propagating modes which can be labeled by a real wave vector $k$ (in general, nonpropagating modes would also carry current and we would have integrate over all frequencies). We then get the following forms for the particle and energy currents:

$$
\begin{gathered}
\left\langle\hat{j}_{l}^{n}\right\rangle=\frac{1}{2 \pi} \int_{0}^{\pi} d k \nu(k) T(k)\left[f-f^{\prime}\right], \\
\left\langle\hat{j}_{l}^{u}\right\rangle=\frac{1}{2 \pi} \int_{0}^{\pi} d k \nu(k) \epsilon(k) T(k)\left[f-f^{\prime}\right],
\end{gathered}
$$

where

$$
\nu(k)=\partial \epsilon(k) / \partial k=2 \sin (k),
$$

which are precisely of the Landauer form.

In order to get the four-probe result we need to find the actual potential and temperature differences across the system. We imagine doing this by putting potentiometers and thermometers at points on the leads ( $A$ and $B$ in Fig. 1). These measure the local particle and energy density on the leads from which one can compute the chemical potential and temperature. We note that we do not expect local thermal equilibration in this noninteracting system and so these are only effective potentials and temperatures.

We start with the general expressions for densities [similar to Eqs. (7)] and after using the various determinantal identities we get (for points $l$ located on the left lead) an integrand which contains a factor $\sin ^{2}\left[k\left(N_{l}-l\right)\right]$. Assuming that $N_{l}$ is large and $l$ is not too close to the point of contact with reservoirs this factor can be replaced by $1 / 2$. We then get for the particle and energy densities

$$
\begin{gathered}
\left\langle\hat{n}_{l}\right\rangle=\frac{1}{2 \pi} \int_{0}^{\pi} d k\left\{[2-T(k)] f+T(k) f^{\prime}\right\}, \\
\left\langle\hat{u}_{l}\right\rangle=\frac{1}{2 \pi} \int_{0}^{\pi} d k \epsilon(k)\left\{[2-T(k)] f+T(k) f^{\prime}\right\} .
\end{gathered}
$$

We get similar expressions for densities at points on the right lead. The expressions in Eqs. (11) and (12) are identical to those obtained from semiclassical arguments, are true for ideal contacts, and lead to the usual four-probe formulas. The results of Eqs. (11) and (12) have been obtained earlier by Tasaki ${ }^{5}$ using the theory of $C^{*}$ algebra. They can be easily extended to the case where the leads are still one dimensional but the system is of more general form. Thus let the system consist of $N_{s}$ points of which 1 and $N_{s}$ are connected to the two leads. Let us specify the system by the matrix $\hat{\phi}$ such that $\hat{\phi}_{l l}=v_{l}-\omega$ and $\hat{\phi}_{l m}=-1$ whenever two distinct points $l$ and $m$ are connected by a hopping element. Then all the above formulas, Eqs. (11) and (12), for currents and densities hold provided we evaluate them within the leads and use the appropriate expression for the transmission coefficient, namely,

$$
T=\frac{4 \sin (k)^{2} F^{2}}{\left|d_{1, N_{s}}-e^{i k}\left(d_{2, N_{s}}+d_{1, N_{s}-1}\right)+e^{i 2 k} d_{2, N_{s}-1}\right|^{2}},
$$




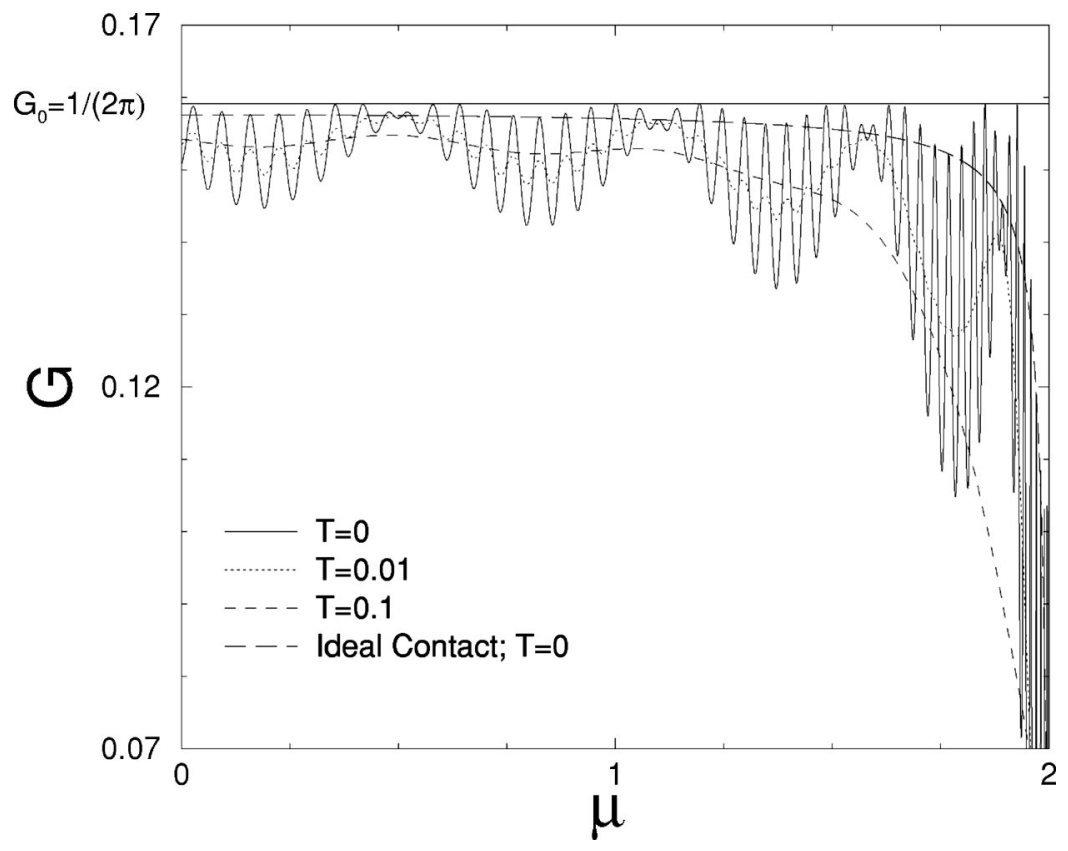

FIG. 2. Plot of conductance vs Fermi level, at three different temperatures $(T)$, of a wire with a single impurity and imperfect contacts. The corresponding plot for perfect contacts at $T=0$ is also shown. The horizontal line is the ideal conductance $G_{0}=1 /(2 \pi)$ (in units of $\left.e^{2} / \hbar\right)$.

where $F$ denotes the determinant of the submatrix formed from $\hat{\phi}$ by deleting the first row and $N_{s}$ th column while $d$ is as before, but now constructed from $\hat{\phi}$.

\section{Application}

As an application we show how the experimental results of Kong et al. ${ }^{8}$ can be understood qualitatively using our results by assuming imperfect contacts.

We consider again semi-infinite ideal reservoirs but make the contacts nonideal by setting $\gamma=\gamma^{\prime}=0.9$. As system we take a wire with a single impurity at site $s$ (thus $v_{s} \neq 0$ ). The linear response conductance is then given by $G$ $=\int_{-\infty}^{\infty} d \omega J(\omega) f(\omega)[1-f(\omega)]$. We evaluate this numerically at different temperatures for $N=100, s=10$, and $v_{s}$ $=0.2$ (Fig. 2). We see the following features: (a) a rapid oscillation of the conductance due to resonances with standing waves in the wire, (b) a slower oscillation due to standing waves formed between boundary and impurity, and (c) a washing away of the oscillations with increasing temperature. These features are qualitatively the same as seen in the experiments in Ref. 8. The overall decrease in conductance with increasing temperature is presumably due to scattering by phonons and hence is not seen here. We have also plotted in Fig. 2 the conductance as given by the usual LF. Note that this does not give the oscillatory features. Thus imperfect contacts cannot be treated as resistances in series with the system. Another rather remarkable effect we see is the enhancement of the conductance as a result of the introduction of imperfect contacts. In fact we can see in Fig. 2 that at certain values of $\mu$ the conductance almost attains the ideal value $1 /(2 \pi)$. Similar features are also obtained if we make the contacts ideal but take other forms of reservoirs (e.g., rings or two-dimensional baths).

\section{Interacting systems}

For this case the present approach readily yields to a perturbative treatment. For illustration consider the case where the Hamiltonian of the system (and lead) $H_{S L}$ contains an interacting part and is given by

$$
H_{S L}=-\sum_{l=1}^{N-1}\left(c_{l}^{\dagger} c_{l+1}+c_{l+1}^{\dagger} c_{l}\right)+\sum_{l=1}^{N} v_{l} c_{l}^{\dagger} c_{l}+\Delta \sum_{l=1}^{N-1} n_{l} n_{l+1},
$$

while the reservoirs are still taken to be noninteracting. In this case, Eqs. (1) take the form

$$
\begin{gathered}
\dot{c}_{1}=i c_{2}-i v_{1} c_{1}-i \Delta n_{2} c_{1}+i \gamma c_{\alpha}, \\
\dot{c}_{l}=i\left(c_{l-1}+c_{l+1}\right)-i v_{l} c_{l}-i \Delta\left(n_{l-1}+n_{l+1}\right) c_{l}, \\
2 \leqslant l \leqslant N-1, \\
\dot{c}_{N}=i c_{N-1}-i v_{N} c_{N}-i \Delta n_{N-1} c_{N}+i \gamma^{\prime} c_{\alpha^{\prime}},
\end{gathered}
$$

and, being nonlinear, can no longer be solved exactly. However, it is straightforward to obtain a perturbative solution which, schematically, has the form $c(\omega)=\hat{Z}^{-1} h$ $-\Delta \hat{Z}^{-1} \int d \omega^{\prime} \int d \omega^{\prime \prime} \hat{Z}^{-1} h \hat{Z}^{-1} h \hat{Z}^{-1} h+O\left(\Delta^{2}\right)$. The operators for particle density and particle current remain unchanged, and we can obtain their expectation values as a perturbation series using this solution. Another possibility would be to solve Eq. (14) using a self-consistent mean-field theory.

\section{HEAT TRANSPORT IN OSCILLATOR CHAINS}

We now use the FKM method to study heat conduction in quantum-disordered harmonic chains connected to general heat reservoirs which are modeled as an infinite collection of oscillators. There has been some earlier work on quantum wires $^{24,25}$ which follows a similar approach but we give a more clear and complete picture and make some interesting predictions for experiments.

As in the electronic case we obtain formal exact expressions for the thermal current and show that, for a special case, they reduce to Landauer-like forms. We also analyze 
the asymptotic system size dependence of the current and show that, depending on the reservoirs, a long wire can behave either like an insulator or a superconductor. Our results should be useful in interpreting recent experiments ${ }^{9}$ on heat transport in insulating nanowires and nanotubes. They are also of interest in the context of the question of validity of Fourier's law in one-dimensional systems, a problem that has received much attention recently. ${ }^{23}$ A large amount of work on classical Hamiltonian systems seems to indicate that Fourier's law is not valid in one-dimensionsal momentumconserving systems. Our work here shows that this is true even in quantum mechanical systems.

\section{A. Formalism and main results}

We consider a mass-disordered harmonic chain containing $N$ particles with the following Hamiltonian:

$$
H=\sum_{l=1}^{N} \frac{p_{l}^{2}}{2 m_{l}}+\sum_{l=1}^{N-1} \frac{\left(x_{l}-x_{l+1}\right)^{2}}{2}+\frac{\left(x_{1}^{2}+x_{N}^{2}\right)}{2},
$$

where $\left\{x_{l}\right\}$ and $\left\{p_{l}\right\}$ are the displacement and momentum operators of the particles and $\left\{m_{l}\right\}$ are the random masses. Sites 1 and $N$ are connected to two heat reservoirs ( $L$ and $R$ ) which we now specify. We model each reservoir by a collection of $M$ oscillators. Thus the left reservoir has the following Hamiltonian:

$$
\begin{aligned}
H_{L} & =\sum_{l=1}^{M} \frac{P_{l}^{2}}{2}+\sum_{l, m} \frac{1}{2} K_{l m} X_{l} X_{m} \\
& =\sum_{s=1}^{M} \frac{\widetilde{P}_{s}^{2}}{2}+\frac{\omega_{s}^{2}}{2} \widetilde{X}_{s}^{2} \\
& =\sum_{s=1}^{M}\left(n_{s}+1 / 2\right) \omega_{s} a_{s}^{\dagger} a_{s},
\end{aligned}
$$

where $K_{l m}$ is a general symmetric matrix for the spring couplings, $\left\{X_{l}, P_{l}\right\}$ are the bath operators, and $\left\{\widetilde{X}_{l}, \widetilde{P}_{l}\right\}$ are the corresponding normal-mode operators. They are related by the transformation $X_{l}=\Sigma_{s} U_{l s} \widetilde{X}_{s}$ where $U_{l s}$, chosen to be real, satisfies the eigenvalue equation $\Sigma_{l} K_{n l} U_{l s}=\omega_{s}^{2} U_{n s}$ for $s=1,2, \ldots, M$. The annihilation and creation operators $a_{s}$ and $a_{s}^{\dagger}$ are given by $a_{s}=\left(\widetilde{P}_{s}-i \omega_{s} \widetilde{X}_{s}\right) /\left(2 \omega_{s}\right)^{1 / 2}$, etc., and $n_{s}=a_{s}^{\dagger} a_{s}$ is the number operator.

The two reservoirs are initially in thermal equilibrium at temperatures $T_{L}$ and $T_{R}$. At time $t=\tau$ the system, which is in an arbitrary initial state is connected to the reservoirs. We consider the case where site 1 on the system is connected to $X_{p}$ on the left reservoir while $N$ is connected to $X_{p^{\prime}}$ on the right reservoir. Thereafter the whole system evolves through the combined Hamiltonian

$$
H_{T}=H+H_{L}+H_{R}-k x_{1} X_{p}-k^{\prime} x_{N} X_{p^{\prime}} .
$$

The Heisenberg equations of motion of the system variables are the following (for $t>\tau$ ):

$$
\begin{gathered}
m_{1} \ddot{x}_{1}=-\left[2 x_{1}-x_{2}\right]+k X_{p}, \\
m_{l} \ddot{x}_{l}=-\left(-x_{l-1}+2 x_{l}-x_{l+1}\right), \quad 1<l<N, \\
m_{N} \ddot{x}_{N}=-\left[-x_{N-1}+2 x_{N}\right]+k^{\prime} X_{p^{\prime}} .
\end{gathered}
$$

We note that they involve the bath variables $X_{p, p^{\prime}}$. However, these can be eliminated and replaced by effective noise and dissipative terms, by using the equations of motion of the bath variables. Consider the equation of motion of the left bath variables. They have the form

$$
\begin{gathered}
\ddot{X}_{n}=-K_{n l} X_{l}, \quad n \neq p, \\
\ddot{X}_{p}=-K_{p l} X_{l}+k x_{1} .
\end{gathered}
$$

This is a linear inhomogeneous set of equations with the solution

$$
\begin{aligned}
X_{n}= & \sum_{l}\left[F_{n l}(t-\tau) X_{l}(\tau)+G_{n l}(t-\tau) \dot{X}_{l}(\tau)\right. \\
& +\int_{\tau}^{\infty} d t^{\prime} G_{n p}\left(t-t^{\prime}\right) k x_{1}\left(t^{\prime}\right),
\end{aligned}
$$

where

$$
\begin{aligned}
& F_{n l}(t)=\theta(t) \sum_{s} U_{n s} U_{l s} \cos \left(\omega_{s} t\right), \\
& G_{n l}(t)=\theta(t) \sum_{s} U_{n s} U_{l s} \frac{\sin \left(\omega_{s} t\right)}{\omega_{s}} .
\end{aligned}
$$

Thus we find that $X_{p}$ (say) appearing in Eq. (17) has the form $X_{p}(t)=h(t)+k \int_{\tau}^{\infty} d t^{\prime} G_{p p}\left(t-t^{\prime}\right) x_{1}\left(t^{\prime}\right)$. The first part, given by $h(t)=\Sigma_{l}\left[F_{p l}(t-\tau) X_{l}(\tau)+G_{p l}(t-\tau) \dot{X}_{l}(\tau)\right.$, is like a noise term while the second part is like dissipation. The noise statistics is easily obtained using the fact that at time $t=\tau$ the bath is in thermal equilibrium and the normal modes satisfy $\left\langle a_{s}^{\dagger}(\tau) a_{s^{\prime}}(\tau)\right\rangle=f\left(\omega_{s}, \beta_{L}\right) \delta_{s s^{\prime}}$. Here $f=1 /\left(e^{\beta \omega}-1\right)$ is the equilibrium phonon distribution and $\langle\hat{O}\rangle=\operatorname{Tr}[\hat{\rho} \hat{O}]$ where $\hat{\rho}$ is the reservoir density matrix and $\operatorname{Tr}$ is over the reservoir degrees of freedom. We define the Fourier transforms $\quad x_{l}(\omega)=(1 / 2 \pi) \int_{-\infty}^{\infty} d t x_{l}(t) e^{i \omega t}, \quad G_{p p}^{+}(\omega)$ $=\int_{-\infty}^{\infty} d t G_{p p}(t) e^{i \omega t}$, and $h(\omega)=(1 / 2 \pi) \int_{-\infty}^{\infty} d t h(t) e^{i \omega t}$. Taking limits $M \rightarrow \infty$ and $\tau \rightarrow-\infty$ we get

$$
\begin{gathered}
X_{p}(\omega)=h(\omega)+k G_{p p}^{+}(\omega) x_{1}(\omega), \\
\left\langle h(\omega) h\left(\omega^{\prime}\right)\right\rangle=I(\omega) \delta\left(\omega+\omega^{\prime}\right), \\
I(\omega)=\frac{f(\omega) b(\omega)}{\pi}, \\
G_{p p}^{+}(\omega)=\sum_{s} \frac{U_{p s}^{2}}{\omega_{s}^{2}-\omega^{2}}-i b(\omega),
\end{gathered}
$$

where 


$$
b(\omega)=\sum_{s} \frac{\pi U_{p s}^{2}}{2 \omega_{s}}\left[\delta\left(\omega-\omega_{s}\right)-\delta\left(\omega+\omega_{s}\right)\right] .
$$

Similarly for the right reservoir we get $X_{p^{\prime}}=h^{\prime}(\omega)$ $+k^{\prime} G_{p^{\prime} p^{\prime}}^{+}(\omega) x_{N}(\omega)$, the noise statistics of $h^{\prime}(\omega)$ being now determined by $\beta^{\prime}$. The left and right reservoirs are independent so that $\left\langle h(\omega) h^{\prime}\left(\omega^{\prime}\right)\right\rangle=0$. We can now obtain the particular solution of Eq. (17) by taking Fourier transforms and plugging in the forms of $h(\omega)$ and $h^{\prime}(\omega)$. We then get

$$
\begin{gathered}
x_{l}(t)=\int_{-\infty}^{\infty} \hat{Z}_{l m}^{-1}(\omega) h_{m}(\omega) e^{i \omega t}, \\
\hat{Z}=\hat{\phi}_{l m}-\hat{A}_{l m},
\end{gathered}
$$

with

$$
\begin{gathered}
\hat{\phi}_{l m}=-\left(\delta_{l, m+1}+\delta_{l, m-1}\right)+\left(2-m_{l} \omega^{2}\right) \delta_{l, m}, \\
\hat{A}_{l m}=\delta_{l, m}\left[k^{2} G_{p p}^{+}(\omega) \delta_{l, 1}+k^{\prime 2} G_{p^{\prime} p^{\prime}}^{+}(\omega) \delta_{l, N}\right], \\
h_{l}(\omega)=k h(\omega) \delta_{l, 1}+k^{\prime} h^{\prime}(\omega) \delta_{l, N} .
\end{gathered}
$$

We can now proceed to calculate the steady-state values of the observables of interest such as the heat current and temperature profile. We first need to find the appropriate operators corresponding to these. To find the current operator $\hat{j}$ we first define the local energy density $u_{l}=p_{l}^{2} / 4 m_{l}$ $+p_{l+1}^{2} / 4 m_{l+1}+1 / 2\left(x_{l}-x_{l+1}\right)^{2}$. Using the current conservation equation $\partial \hat{u} / \partial t+\partial \hat{j} / \partial x=0$ and the equations of motion we then find that $\hat{j}_{l}=\left(\dot{x}_{l} x_{l-1}+x_{l-1} \dot{x}_{l}\right) / 2$. The steady-state current can now be computed by using the explicit solution in Eq. (4). We get

$$
\begin{aligned}
\left\langle\hat{j}_{l}\right\rangle= & \int_{-\infty}^{\infty} d \omega(i \omega)\left[k^{2} \hat{Z}_{l, 1}^{-1}(\omega) \hat{Z}_{l-1,1}^{-1}(-\omega) I(\omega)\right. \\
& \left.+k^{\prime 2} \hat{Z}_{l, N}^{-1}(\omega) \hat{Z}_{l-1, N}^{-1}(-\omega) I^{\prime}(\omega)\right] .
\end{aligned}
$$

The matrix $Z$ is tridiagonal and using some of its special properties (see Sec. II A) we can reduce the current expression to the following simple form:

$$
\begin{aligned}
\left\langle\hat{j}_{l}\right\rangle & =\frac{k^{2} k^{\prime 2}}{\pi} \int_{-\infty}^{\infty} d \omega \frac{\omega b(\omega) b^{\prime}(\omega)}{\left|Y_{1, N}\right|^{2}}\left(f-f^{\prime}\right) \\
& =\int_{-\infty}^{\infty} d \omega J(\omega)\left(f-f^{\prime}\right),
\end{aligned}
$$

where $J(\omega)=k^{2} k^{\prime 2} \omega b(\omega) b^{\prime}(\omega) / \pi\left|Y_{1, N}\right|^{2}$ has the physical interpretation as the total heat current in the wire due to all right-moving (or left-moving) scattering states of the full Hamiltonian (system + reservoirs). Such scattering states can be obtained by evolving initial unperturbed states of the reservoirs with the full Hamiltonian (see end of Sec. II A). As before we have denoted by $Y_{l, m}$ the determinant of the submatrix of $\hat{Z}$ beginning with the $l$ th row and column and ending with the $m$ th row and column. Similarly let $D_{l, m}$ denote the determinant of the submatrix formed from $\hat{\Phi}$.

\section{B. Ideal reservoirs and contacts: The Landauer case}

For the special case when the reservoirs are also onedimensional chains with nearest-neighbor spring constants $K_{l m}=1$ and the coupling constants $k, k^{\prime}$ are set to unity, we have $G_{p p}^{+}=G_{p^{\prime} p^{\prime}}^{+}=e^{-i k}$, where $\omega=2 \sin (k / 2)$ and $I(\omega)$ $=f(\omega) \sin (k) / \pi$ for $|\omega|<2$ and $I(\omega)=0$ for $|\omega|>2$. In this case Eq. (23) simplifies further and has an interpretation in terms of transmission coefficients of plane waves across the disordered system. We get

$$
J=\frac{1}{4 \pi} \int_{-2}^{2} d \omega \omega\left|t_{N}(\omega)\right|^{2}\left(f-f^{\prime}\right),
$$

where

$$
\left|t_{N}(\omega)\right|^{2}=\frac{4 \sin ^{2}(k)}{\left|D_{1, N_{s}}-e^{i k}\left(D_{2, N_{s}}+D_{1, N_{s}-1}\right)+e^{i 2 k} D_{2, N_{s}-1}\right|^{2}}
$$

is the transmission coefficient at frequency $\omega$. We have thus obtained the Landauer formula ${ }^{2}$ for phononic transport. It is only in this special case of a one-dimensional reservoir and perfect contacts that we get the Landauer formula. The reason is that only in this case is the transmission through the contacts perfect, and this requirement is one of the crucial assumptions in the Landauer derivation. Note that in Eq. (24) (i) the transmission coefficient does not depend on bath properties and (ii) transmission is only through propagating modes. For general reservoirs where we need to use Eq. (23) the factor $J(\omega)$ involves not just the properties of the wire but also the details of the spectral functions of the reservoirs. Thus the conductivity of a sample can show a rather remarkable dependence on reservoir properties as we shall see below. The above Landauer-like formula has earlier been stated in Ref. 26 and derived more systematically in Ref. 27. We note that in the high temperature limit $T, T^{\prime} \rightarrow \infty$, Eq. (24) reduces to the classical limit obtained exactly in Refs. 2022.

\section{Asymptotic system-size dependences}

In the case of electrical conduction the conductance of a long disordered chain decays exponentially with system size as a result of localization of states. In the case of phonons the long-wavelength modes are not localized and can carry current. This leads to power-law dependences of the current on system size as has been found earlier in the context of heat conduction in classical oscillator chains. A surprising result is that the conductivity of such disordered chains depends not just on the properties of the chain itself but also on those of the reservoirs to which it is connected. It can be shown ${ }^{22}$ that the asymptotic properties of the integral in Eq. (23) depend on the low-frequency $\left(\omega \leqslant 1 / N^{1 / 2}\right)$ properties of the integrand. This means that we will get the same behavior as in the classical case. We summarize some of the main results.

(i) The classical case where the reservoirs are themselves one dimensional. In this case we put $k=k^{\prime}=1$ and the spectral function $G_{p p}^{+}=G_{p^{\prime} p^{\prime}}^{+}=e^{-i k}$ where $\omega=2 \sin (k / 2)$. This 
was treated by Rubin and $\mathrm{Greer}^{21}$ and it was found that $J$ $\sim 1 / N^{1 / 2}$. Thus the ideal Landauer case will also show this behavior.

(ii) The case of reservoirs which give $\delta$-correlated Langevin noise corresponds to taking $k=k^{\prime}=1$ and $G_{p p}^{+}=G_{p^{\prime} p^{\prime}}^{+}$ $=-i \gamma \omega$. The classical case was first treated by Casher and Lebowitz ${ }^{28,20}$ and one gets $J \sim 1 / N^{3 / 2}$.

(iii) In general one gets $J \sim 1 / N^{\alpha}$ where $\alpha$ depends on the low-frequency behavior of the spectral functions $G_{p p}(\omega)$ and $G_{p^{\prime} p^{\prime}}\left(\omega^{\prime}\right){ }^{22}$

Note that the case $\alpha<1$ leads to infinite thermal conductivity while $\alpha>1$ gives a vanishing conductivity. Thus, depending on the properties of the heat baths, the same wire can show either superconducting or insulating behavior. The usual Fourier's law would predict $J \sim 1 / N$, independent of reservoirs. Thus Fourier's law is not valid in quantum harmonic chains, even in the presence of disorder. This breakdown of Fourier's law in 1D systems has been noted in a number of earlier studies on classical systems ${ }^{23}$ which have looked at the effects of scattering both due to impurities and nonlinearities.

\section{Temperature profiles}

The local temperature of a particle can be determined from its average kinetic energy $k e_{l}=\left\langle p_{l}^{2} /\left(2 m_{l}\right)\right\rangle$. We get

$$
\begin{aligned}
k e_{l}= & \frac{1}{2} \int_{-\infty}^{\infty} d \omega m_{l} \omega^{2}\left[k^{2} \hat{Z}_{l, 1}^{-1}(\omega) \hat{Z}_{l, 1}^{-1}(-\omega) I(\omega)\right. \\
& \left.+k^{\prime 2} \hat{Z}_{l, N}^{-1}(\omega) \hat{Z}_{l, N}^{-1}(-\omega) I^{\prime}(\omega)\right] .
\end{aligned}
$$

This is straightforward to evaluate numerically for given systems and reservoirs. For the special case of heat transmission through a perfect one-dimensional harmonic chain attached to one-dimensional reservoirs through perfect contacts (i.e., $k=k^{\prime}=1$ ), Eq. (25) simplifies (for large $N$ ) to

$$
k e_{l}=\frac{1}{8 \pi} \int_{-\pi}^{\pi} d k \omega_{k}\left[f\left(\omega_{k}\right)+f^{\prime}\left(\omega_{k}\right)\right],
$$

where $\omega_{k}=2 \sin (k / 2)$. For $T=T^{\prime}$, we get

$$
k e_{l}=\frac{1}{4 \pi} \int_{0}^{\pi} d k \omega_{k} \operatorname{coth}\left(\frac{\beta \omega_{k}}{2}\right),
$$

which is the expected equilibrium kinetic energy density on an infinite chain. For weak coupling to the reservoirs, which can be achieved by making $k$ and $k^{\prime}$ small, we expect that the energy density profile for the system should correspond to that of a finite chain. We verify this numerically by evaluating Eq. (25) for $k=k^{\prime}=0.1$ and $T=T^{\prime}$ (Fig. 3). We compare this with the equilibrium kinetic energy profile of a finite chain given by

$$
k e_{l}=\frac{1}{4} \sum_{s} \omega_{s} \operatorname{coth}\left(\frac{\beta \omega_{s}}{2}\right) \psi_{s}^{2}(l),
$$

where $\quad \psi_{s}(l)=[2 /(N+1)]^{1 / 2} \sin (k l)$ and $\omega_{s}=2 \sin (k / 2)$, where $k=s \pi /(N+1)$, with $s=1,2, \ldots, N$. Note that unlike

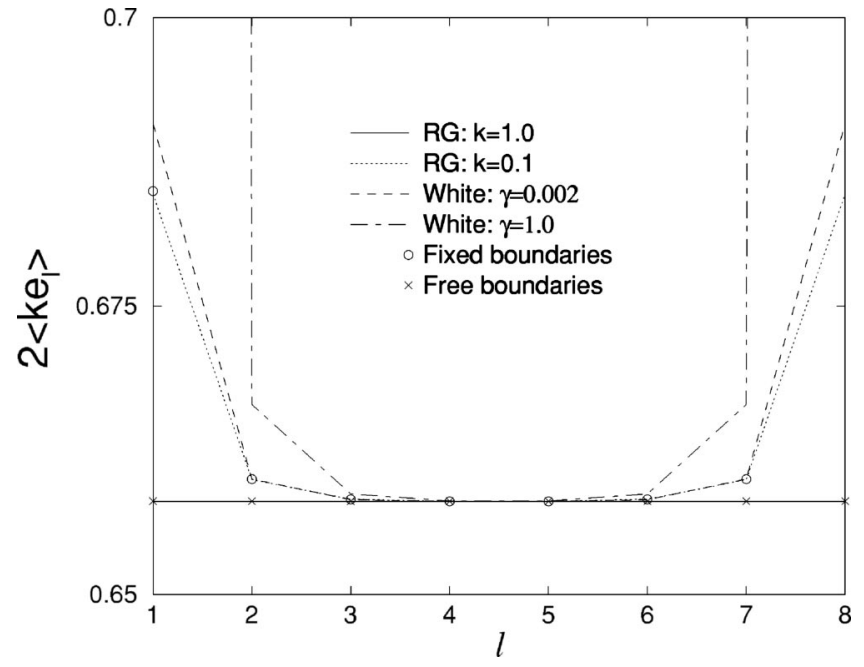

FIG. 3. Kinetic energy density profile in a pure harmonic chain $(N=8)$ attached to reservoirs at equal temperatures $T=T^{\prime}=0.2$. Two different kinds of reservoirs are considered: one-dimenional reservoirs (RG) and $\delta$-correlated noise reservoirs (white). The exact equilibrium density profiles for an infinite chain (free) and one with fixed ends are also given.

in the classical case where the energy density is a constant, in the quantum case, this is not always true. It is instructive to look at the equilibrium properties for the case where the driving is by a $\delta$-correlated noise [case (ii) discussed earlier]. In this case the weak-coupling limit corresponds to taking the damping constant $\gamma \ll 1$. The temperature profiles obtained from Eq. (25) for two different values of $\gamma$ are plotted in Fig. 3.

We now consider temperature profiles in the nonequilibrium case $\left(T \neq T^{\prime}\right)$. For the Rubin-Greer (or Landauer case, i.e., 1D reservoirs, perfect contacts), at high temperatures the local temperature is given by $T_{l}=2 k e_{l}$ and from Eq. (26) we get $T_{l}=\left(T+T^{\prime}\right) / 2$ which is the classical result. ${ }^{29}$ At low temperatures and imperfect contacts $k, k^{\prime} \neq 1$ we evaluate the local kinetic energy profile numerically using Eq. (25). As can be seen in Fig. 4 the temperature in the bulk still has the same constant value. At the boundaries, however, we see a curious feature noted earlier by Refs. 24 and 29: the temperature close to the hot end is lower than the average temperature while that at the colder end is higher than the average. For the case with $\delta$-correlated noise, at high temperatures, we recover the temperature profiles obtained ealier for classical chains in Ref. 29. At low temperatures we get results similar to those found by Zurcher and Talkner ${ }^{24}$ and there seem to be some qualitative differences from the classical temperature profiles, depending on the value of $\gamma$.

\section{DISCUSSION}

We note that the more popular approach of treating open quantum systems is the Caldeira-Leggett formulation. In that approach, one deals with density matrices and the treatment becomes complicated. In the context of the present problem one is not really interested in the full distribution but rather in physical observables like the steady-state currents and 


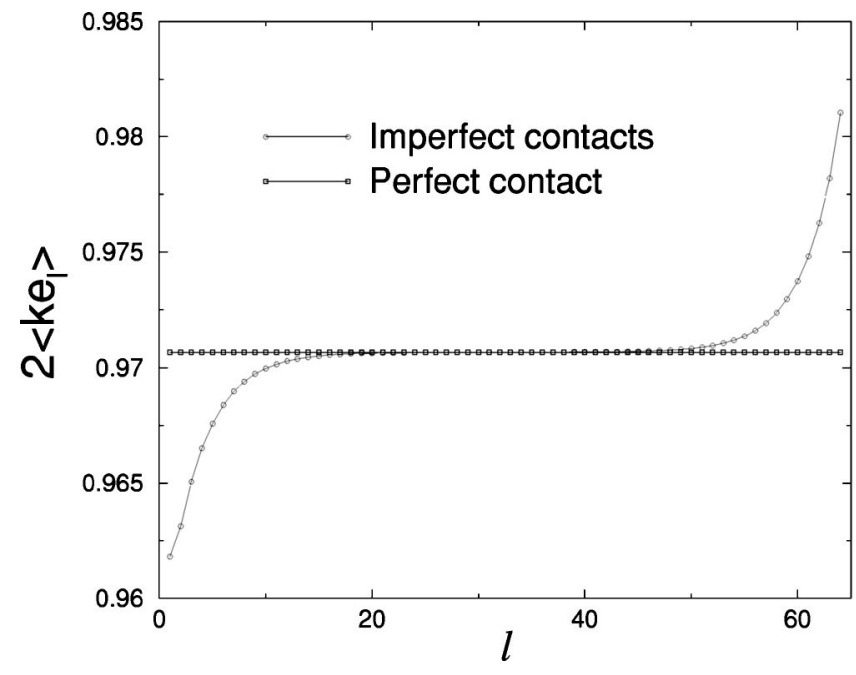

FIG. 4. Kinetic energy density profile in a pure harmonic chain $(N=64)$ attached to one-dimensional reservoirs at temperatures $T$ $=1.0$ (left) and $T^{\prime}=0.5$ (right), for perfect and imperfect $\left(k=k^{\prime}\right.$ $=0.9$ ) contacts. The temperatures considered are not very high, and so the bulk temperature is different from the classically expected value $T_{a v}=0.75$.

densities and these are basically second moments of the distribution. The FKM formulation is then more appropriate and for linear systems one can get exact results. The other approach of treating nonequilibrium systems which has been used quite extensively in the mesoscopic context is the Keldysh formalism. This is a perturbative treatment where one writes equations of motion for a set of Green functions and relates them to self-energies through the Dyson equations. The current is expressed in terms of these Green functions. In special cases the Dyson equations can be solved exactly and indeed some of our results can be obtained. 3,14,30 On the other hand, our method is more transparent and direct. We integrate out the reservoir degrees of freedom to get effective Langevin-type equations of motion for the system. These are solved and quickly lead to useful results on currents and densities of both particle and heat which are automatically expressible in terms of unperturbed Green functions. The connection to scattering theory is also immediate and explicit. Finally one obtains a nice physical picture of the reservoirs serving as effective sources of noise and dissipation. Note that our approach makes connections between different approaches such as the Caldeira-Legett, Keldysh, scattering theory, and the transfer-Hamiltonian methods.

The FKM formulation was earlier used in studying heat transport in classical disordered harmonic chains, and it is particularly nice that the method can be extended to the quantum mechanical regime. Earlier results on classical chains are then obtained as limiting cases. The more general quantum mechanical results can be expressed in forms where one can see connections with other approaches such as Landauer, Keldysh, etc.

The dependence of transport properties of a system on the reservoir properties is at first glance a surprsing fact and we briefly comment on this. From our usual experience in the macroscopic world, one usually thinks of the conductivity of a system as an intrinsic property, not dependent on the properties of reservoirs. Imagine making a measurement of the thermal conductivity of a wire by putting its ends in contact with heat baths at two different temperatures and measuring the resulting current. The normal expectation is that the answer should not depend on the material properties of the heat baths. And indeed this expectation holds true quite often. One physical way of understanding this is that, as long as the system (the wire) is a strongly interacting system, with good ergodicity properties, then one can expect that, soon after contact is made with the reservoirs, the ends of the wire would reach a state of local thermal equilibrium with the reservoirs. This local equilibrium would be completely determined by just the temperature of the reservoir and this then drives the current in the wire. In the mesoscopic domain, however, there are situations when the interactions between the carriers are not strong enough to let the system reach local equilibrium. And then one finds that the conducting properties of a wire is no longer intrinsic to the wire but depends on details of the reservoirs. Thus any calculation of transport properties would require a detailed modeling of the reservoirs. An explicit demonstration of the conditions under which reservoir dependence goes away does not seem to exist at present.

As has been shown here the FKM method works as easily for both electronic transport in disordered fermionic wires and thermal transport in disordered harmonic chains. In both cases we are able to obtain exact formal expressions for particle and thermal currents and these have very similar forms. Both depend on details of the reservoir spectral functions. The usual Landauer case where one writes the current in terms of transmission factor of one-dimensional plane waves is shown to follow, exactly, for the choice of onedimensional reservoirs and perfect contacts. In general, however, one needs to use modified Landauer formulas and this can be quite crucial in interpreting experimental data. For example we have shown that the oscillations in conductance seen in the experiments by Kong et al. cannot be explained unless the contacts and reservoirs are treated quantum mechanically. We also find the rather counterintuitive prediction that imperfect contacts can enhance the conductance of a wire. In the phonon case we make a couple of predictions that are interesting from the experimental point of view: (i) the large-system-size behavior of the heat current is a power law and the power depends on reservoir properties, and (ii) temperature profiles in perfect wires show somewhat counterintuitive features close to contacts. It would be interesting to see if our predictions, which are true for strictly onedimensional chains, can be verified in experiments on nanowires.

\section{ACKNOWLEDGMENTS}

We are grateful to N. Kumar, H. Mathur, T. V. Ramakrishnan, and A. K. Raychaudhuri for useful comments. B.S.S. was supported in part by Indo French Grant No. IFCPAR/ 2404.1. A.D. acknowledges support from the National Science Foundation under Grant No. DMR 0086287. 
*On leave from Raman Research Institute, Bangalore, India.

${ }^{\dagger}$ Also at JNCASR, Bangalore, India.

${ }^{1}$ J. Bardeen, Phys. Rev. Lett. 6, 57 (1961).

${ }^{2}$ R. Landauer, IBM J. Res. Dev. 1, 223 (1957).

${ }^{3}$ C. Caroli et al., J. Phys. C 4, 916 (1971).

${ }^{4}$ G. Y. Hu and R. F. O'Connell, Phys. Rev. B 36, 5798 (1987).

${ }^{5}$ S. Tasaki, Chaos, Solitons Fractals 12, 2657 (2001).

${ }^{6}$ T. N. Todorov, G. A. D. Briggs, and A. P. Sutton, J. Phys.: Condens. Matter 5, 2389 (1993).

${ }^{7}$ C. T. White and T. N. Todorov, Nature (London) 393, 240 (1998); S. J. Tans et al., ibid. 386, 474 (1997); A. Bachtold et al., Phys. Rev. Lett. 84, 6082 (2000).

${ }^{8}$ J. Kong et al., Phys. Rev. Lett. 87, 106801 (2001).

${ }^{9}$ K. Schwab et al., Nature (London) 404, 974 (2000); T. S. Tighe, J. M. Worlock, and M. L. Roukes, Appl. Phys. Lett. 70, 2687 (1997); P. Kim et al., Phys. Rev. Lett. 87, 215502 (2001).

${ }^{10}$ M. Buttiker et al., Phys. Rev. B 31, 6207 (1985); U. Sivan and Y. Imry, ibid. 33, 551 (1986); H. L. Engquist and P. W. Anderson, ibid. 24, 1151 (1981); E. N. Economou and C. M. Soukoulis, Phys. Rev. Lett. 46, 618 (1981); P. W. Anderson et al., ibid. 22, 3519 (1980); Y. Imry, in Directions in Condensed Matter Physics, edited by G. Grinstein and G. Mazenko (World Scientific, Singapore, 1986); D. C. Langreth and E. Abrahams, Phys. Rev. B 24, 2978 (1981); D. S. Fisher and P. A. Lee, ibid. 23, 6851 (1981).

${ }^{11}$ Yu. V. Sharvin, Sov. Phys. JETP 21, 655 (1965).

${ }^{12}$ A. Szafer and A. D. Stone, Phys. Rev. Lett. 62, 300 (1989).

${ }^{13}$ Y. Imry and R. Landauer, Rev. Mod. Phys. 71, S306 (1999).

${ }^{14}$ Y. Meir and N. S. Wingreen, Phys. Rev. Lett. 68, 2512 (1992).

${ }^{15}$ G. W. Ford, M. Kac, and P. Mazur, J. Math. Phys. 6, 504 (1965).
${ }^{16}$ G. W. Ford, J. T. Lewis, and R. F. O'Connell, Phys. Rev. A 37, 4419 (1988).

${ }^{17}$ A. O. Caldeira and A. J. Leggett, Ann. Phys. (N.Y.) 149, 374 (1983).

${ }^{18}$ L. V. Keldysh, Sov. Phys. JETP 20, 1018 (1965).

${ }^{19}$ A. N. Cleland, J. M. Schmidt, and J. Clarke, Phys. Rev. B 45, 2950 (1992); G. Y. Hu and R. F. O'Connell, ibid. 49, 16505 (1994); 46, 14219 (1992).

${ }^{20}$ A. J. O'Connor and J. L. Lebowitz, J. Math. Phys. 15, 692 (1974).

${ }^{21}$ R. Rubin and W. Greer, J. Math. Phys. 12, 1686 (1971).

${ }^{22}$ A. Dhar, Phys. Rev. Lett. 86, 5882 (2001).

${ }^{23}$ F. Bonetto, J. L. Lebowitz, and L. Rey-Bellet, math-ph/0002052 (unpublished); A. Dhar, Phys. Rev. Lett. 86, 3554 (2001); P. Grassberger, W. Nadler, and L. Yang, ibid. 89, 180601 (2002); P. Grassberger and L. Yang, cond-mat/0204247 (unpublished); G. Casati and T. Prosen, Phys. Rev. E 67, 015203 (2002); B. Li, H. Zhao, and B. Hu, Phys. Rev. Lett. 86, 63 (2001); A. V. Savin, G. P. Tsironis, and A. V. Zolotaryuk, ibid. 88, 154301 (2002); S. Lepri, R. Livi, and A. Politi, ibid. 78, 1896 (1997); O. Narayan and S. Ramaswamy, ibid. 89, 200601 (2002).

${ }^{24}$ U. Zurcher and P. Talkner, Phys. Rev. A 42, 3278 (1990).

${ }^{25}$ K. Saito, S. Takesue, and S. Miyashita, Phys. Rev. E 61, 2397 (2000); A. Ozpineci and S. Ciraci, Phys. Rev. B 63, 125415 (2001); K. R. Patton and M. R. Geller, ibid. 64, 155320 (2001).

${ }^{26}$ L. G. C. Rego and G. Kirczenow, Phys. Rev. Lett. 81, 232 (1998).

${ }^{27}$ M. P. Blencowe, Phys. Rev. B 59, 4992 (1999).

${ }^{28}$ A. Casher and J. L. Lebowitz, J. Math. Phys. 12, 1701 (1971).

${ }^{29}$ Z. Rieder, J. L. Lebowitz, and E. Lieb, J. Math. Phys. 8, 1073 (1967).

${ }^{30}$ S. Hershfield, J. H. Davies, and J. W. Wilkins, Phys. Rev. B 46, 7046 (1992). 